\title{
Operability Evaluation of Pleasure Boats Based on Usability as Addressed by ISO 9241-11*
}

\author{
Shigehiro TOYAMA**, Kohei IIHAMA**, Fujio IKEDA**, \\ Hiroaki SETA*** and Nobuo EZAKI*** \\ ${ }^{* *}$ Nagaoka National College of Technology \\ 888 Nishikatakai-machi, Nagaoka-shi, Niigata, Japan \\ E-mail:toyama@nagaoka-ct.ac.jp \\ *** Toba National College of Maritime Technology \\ 1-1 Ikegami-cho, Toba-shi, Mie, Japan
}

\begin{abstract}
This paper proposes an evaluation method for operability of pleasure boats in order to develop an electronic control steering system of those boats. Based on the concept of usability as addressed by ISO 9241-11, some evaluation standards such as effectiveness and efficiency are defined for a task of avoiding collision and resuming original course. For safety and repeatability, the simulation with a simplified ship simulator, which is composed a manual hydraulic steering system as hardware in HILS, is performed instead of experiments. As the results of the simulation, it is found that the operability of the manual hydraulic steering system deteriorates in the case of emergency collision avoidance. In particular, the wakes of the course resuming after the emergency collision avoidance are caused to be winding by wrong estimate of the normal pressure and by time delay in the dynamics of the pleasure boat. Therefore, it is found that the electronic control steering system of pleasure boats should have the functions to support estimation of the normal pressure and to compensate the time delay.
\end{abstract}

Key words: Ship, Pleasure Boat, Operability, Usability, Man-Machine Interface, Ship Simulator

\section{Introduction}

The number of maritime accidents of pleasure boats has a trend to increase in recent years ${ }^{(1)}$. The background is supposed that beginner pilots who are insufficient of elementary knowledge and skills are increasing, since marine leisure has rapidly and widely spread under high desire of Japanese people for leisure. It is also supposed that ever changing operation situation and complicated maneuverability, which is caused by nonlinear hydrodynamic force, could induce inappropriate operation that is one of the causes for the maritime accidents. Manual hydraulic steering systems are widely used for pleasure boats. If especially beginner captains steer pleasure boats, it is necessary to secure good operability of steering system. However, it is hard to say that the operability of manual hydraulic steering systems is superior. One of the reasons is that reaction torque of the helm through steering system is not so rich that captains can feel the magnitude of normal pressure at the rudder. Another reason is not so quick steering, since the helm lock to lock is generally 4-7 turns. In order to improve the operability, By-Wire technique ${ }^{(2),(3)}$ should be introduced like Fly-By-Wire system of airplane which communicate the pilot's steering information with electric signal ${ }^{(4)}$, and advanced steering control system of pleasure boats should be constructed like flight control system to prevent malfunction and stabilize posture Eng., Vol.79, No.801, C (2013), pp.1415-1426 (Received 13 Oct., 2012) [DOI: 10.1299/jsdd.7.378]

Copyright $\odot 2013$ by JSME 
for airplanes ${ }^{(5),(6)}$ and helicopters ${ }^{(7)}$. For development of such steering control system to realize universal operability for pleasure boats, the user interface should be improved with the concept of human centered design. Although various studies on evaluation method for maneuverability of airplanes have been reported ${ }^{(8),(9)}$, Pilot Rating mainly contributes to development of airplanes with superior maneuverability ${ }^{(10)}$. Although the evaluation method of maneuverability for automobiles is not necessarily established, various methods such as using Pilot Rating as the model, evaluating task performance, and measuring driver's mental and physical responses have been studied ${ }^{(11)}$. However, the evaluation method of operability for pleasure boats has not been seen as far as the authors know.

In this paper, an operability evaluation method for steering systems of pleasure boats based on usability addressed by ISO 9241-11 is proposed. ISO9241-11 defines that usability is degree to which specified users can achieve specified goals in a particular environment with effectiveness, efficiency and satisfaction. Based on this definition, an operation simulation task to evaluate the operability is set, and some evaluation items of effectiveness and efficiency are proposed. The proposed method based on usability can considered as an evaluation method, which implies standpoints of Pilot rating and task performance evaluation for airplanes and automobiles and also implies the concept of human centered design. The operation simulations are formed from a simplified ship simulator, which can execute high safety and reproducible simulations. A hydraulic manual steering mechanism is evaluated by the proposed items, and some design criteria of the electronic control steering system ${ }^{(3)}$ for pleasure boats are finally investigated.

\section{Manual Hydraulic Steering System}

Figure 1 shows an outline of a typical hydraulic steering system, which is widely employed for pleasure boats. The system mainly consists of a helm, a helm pump, a hydraulic cylinder and a rudder mechanism. When captains turn the helm, hydraulic oil is ejected by the helm pump, which is directly connected to the helm. When the hydraulic oil flows into the hydraulic cylinder, the rudder is revolved by the piston rod. Turning moment with respect to the center of gravity for the hull arises, when revolution of the rudder generates the normal pressure $P_{N}$ as shown in Fig. 2. The normal pressure $P_{N}$ is generally known as follows ${ }^{(12)}$.

$$
P_{N}=\frac{\rho}{2} \cdot A_{R} \cdot f_{\alpha} \cdot V^{2} \cdot \sin \delta,
$$

where $\rho, A_{R}$, and $f_{\alpha}$ show the fluid density, the area of the rudder and the coefficient of the normal pressure. $V$ and $\delta$ show the fluid velocity around the rudder, which changes according to the propeller revolutions at the time of steady operation, and the rudder angle. Note that the normal pressure $P_{N}$ shows nonlinear characteristics not only on the propeller revolutions but also on the rudder angle as shown in Eq. (1). Considering the nonlinear hydraulic force effecting into hull of pleasure boat, captains need to control the nonlinear normal pressure in order to advance toward their intended course.

It is hard to say that the manual hydraulic steering system has satisfactory operability. One reason is that the helm lock to lock is generally set 4-7 turns, which can let captains mistake the neutral position of the helm although it can relieve the operation effort as the lock to lock increases. Another reason is that there is naturally limit of response for emergency cases such as collision avoidance, since captains, who are human beings, serve as a kind of power source of the helm pump. The normal pressure $P_{N}$ is hardly changed by small propeller revolutions and small rudder angle as shown in Eq. (1). And rudder shapes are designed so that the normal pressure $P_{N}$ occurs around the rudder rotation axis in order 
to reduce the operation effort. Those are why there is also situation that the reaction torque of the helm through the hydraulic steering system is remarkably poor. It is extremely hard to estimate the hull motion from reaction torque of the helm thorough the hydraulic steering system, and especially beginner captains who cannot master the maneuverability of pleasure boats sufficiently, could give inappropriate operation. Even though expert captains, their rapid operation could bring excessive rolling angle that might cause capsizal under strong wind-tidal current.

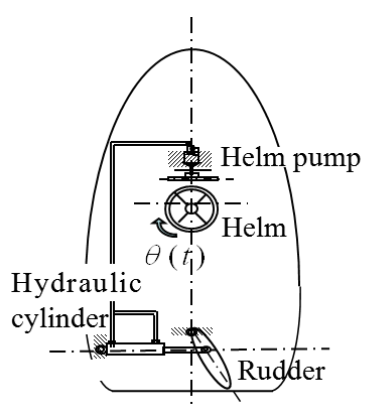

Fig. 1 Manual hydraulic steering system

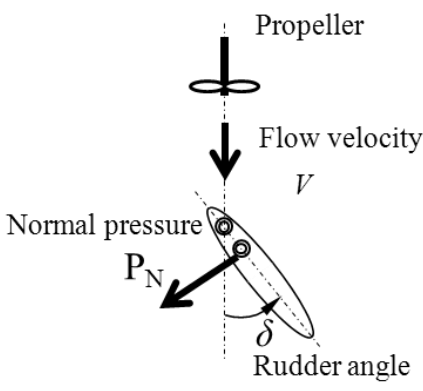

Fig. 2 Normal pressure $P_{N}$

\section{Simplified Ship Simulator}

Figure 3 (a) shows the components of the simplified ship simulator programmed a task to evaluate the operability of steering systems. This ship simulator composes HILS (Hardware In the Loop Simulation), which is a technique used the development and test of complex real-time embed system. As the hardware of the HILS, manual hydraulic steering system such as a helm pump (NE1073-00, Nippatsu Telefrex Inc.), a hydraulic cylinder (NE1009-00, Nippatsu Telefrex Inc.) and a rudder mechanism are employed as shown in Fig. 3 (b),(c). The helm lock to lock is 5.4 turns. As the software of the HILS, the simulation

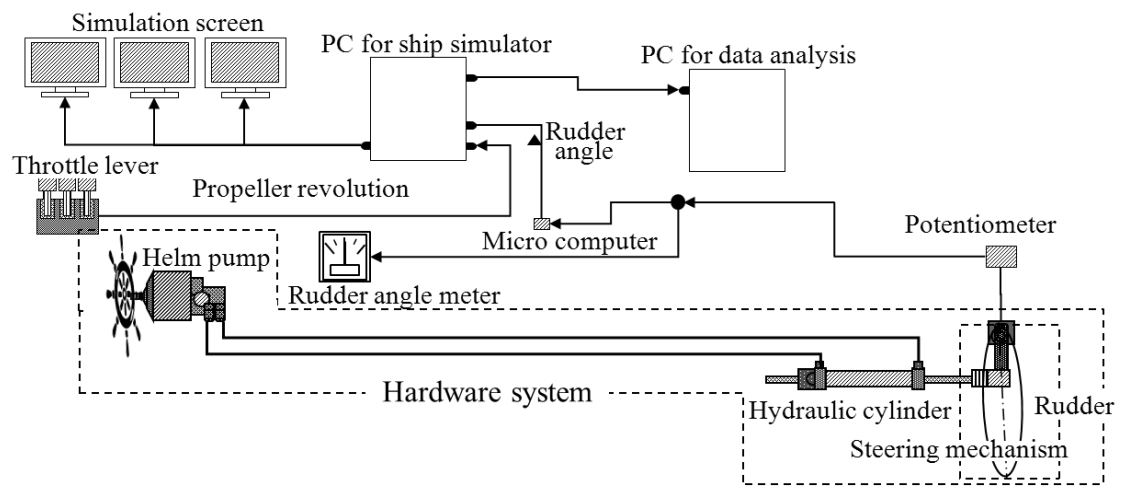

(a) Components

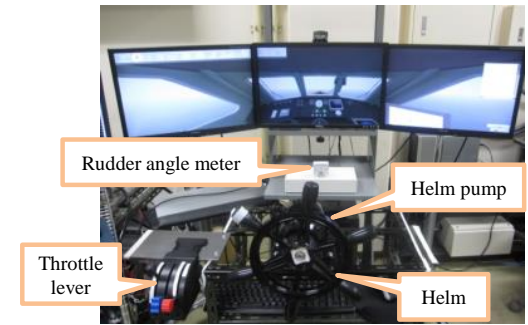

(b) Steering mechanism

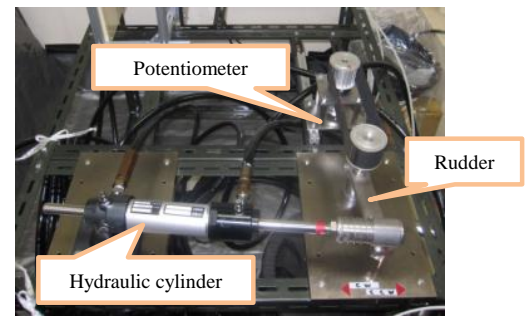

(c) Hydraulic cylinder and rudder mechanism

Fig. 3 Simplified ship simulator 
software "NAUTIS" from VSTEP, which is widely used in voyage training, is installed. The software can output some simulation data such as the 6 DOF ship motions, the rudder angle and the propeller revolution in real-time. The measurement signal of the rudder angle with the potentiometer is sent to the rudder angle meter and to the simulation PC. Also, the propeller revolution signal with the throttle lever is sent to the simulation PC. Then, the simulation software "NAUTIS" calculates the ship motion corresponding to those signals. Based on the computation, the ship motion is described on the three monitors from a view point of the captain. This simplified ship simulator can execute high safety and reproducible simulations.

\section{Operability Evaluation Based on Usability}

\subsection{Task of Operation Simulation}

By ISO9241-11, usability is defined as degree to which specified users can achieve specified goals in a particular environment with effectiveness, efficiency and satisfaction. Based on the definition, the simulation task is set avoiding an obstacle and resuming the original course safely and urgently in poor visibility of a dense fog as shown in Fig. 4. Although various voyage situations can be assumed, such task is considered in order to evaluate transient and steady behavior of captains on whole. As an obstacle, a large vessel, whose total length is $82[\mathrm{~m}]$ and total width is $16[\mathrm{~m}]$, is arranged at $500[\mathrm{~m}]$ point from the starting position, and the end of the evaluation area is arranged as a line at $1000[\mathrm{~m}]$ from the starting point. Captains cannot notice for a short while from the start, since the course is covered with the dense fog. The urgency of avoidance can be variously adjusted by changing the denseness of the fog. The helm revolution angle $\theta(t)$ is defined as shown in Fig. 1, and the course $\psi(t)$ and the rolling angle $\phi(t)$ are defined as shown in Fig. 5.

After noticing the obstacle, captains are demanded to avoid it and to resume the original course quickly and safely. Evaluated more clearly, the effectiveness and the efficiency in the task is quantitated by dividing into the veering period to avoid collision (Phase 1) and the veering period to resume the original course (Phase 2). The final evaluation is determined by considering the results for each phase. As quantitative items of usability, the objective complete time, the number of errors and the operation quantity are typically measured and absolutely or relatively evaluated ${ }^{(13)}$. Some items of the effectiveness and the efficiency in each phase are defined in Section 4.2 and 4.3 respectively, and satisfaction is evaluated by a questionnaire answer after the operation simulation.

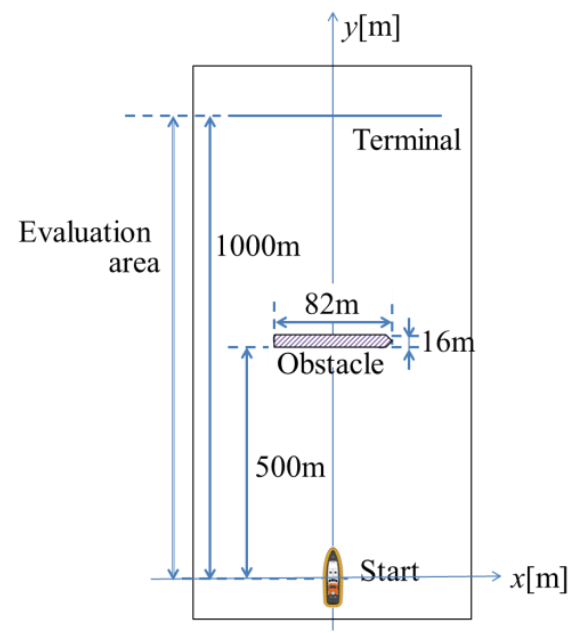

Fig. 4 Outline of task course

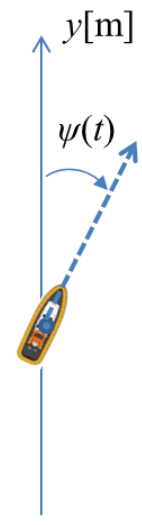

(a) Course

Fig. 5 Evaluated motion of pleasure boat 


\subsection{Veering Period to Avoid Collision (Phase 1)}

Figure 6 shows an outline of the veering situation to avoid the obstacle. As shown in the circled digit one of Fig. 6, the starting moment is assumed to be $t=0$. As shown in the circled digit two, the moment at finding the obstacle while the pleasure boat advanced straightly after the start is assumed to be $t=t_{f}$. As shown in the circled digit three, the complete avoidance moment is assumed to be $t=t_{a}$. Avoiding the obstacle is judged when the course $\psi(t)$ is identical to $\psi_{a}(t)$, which is the angle with the segment $O_{g}-O_{e}$ for the $y$ axis as shown in Fig. 6.

The effectiveness to avoid the obstacle quickly and safely in Phase 1 is quantified with two items, which are the collision risk rate $C L$ on response of avoiding collision and the capsize risk rate $C P_{1}$ on safety. The collision risk rate $C L$ is defined as a rate of the complete avoidance time $t_{a}-t_{f}$ to the predicted time to collision (TTC) at the moment that captains find the obstacle, because the worth of the absolute quantity of the complete time can be changed by difference for the number of propeller revolution and the hull speed. The predicted time to collision is considered as a basis for the relative evaluation of the complete time to difference of the hull speed.

$$
\begin{aligned}
C L & =\frac{(\text { Complete avoidance time })}{(\text { Predicted time to collision at moment of finding obstacle })} \times 100 \\
& =\frac{(\text { Complete avoidance time })}{(\text { Distance to obstacle at finding moment }) /(\text { Boat speed at finding moment })} \times 100 \\
& =\frac{t_{a}-t_{f}}{\left\{500-y\left(t_{f}\right)\right\} / v\left(t_{f}\right)} \times 100
\end{aligned}
$$

The capsize risk rate $C P_{1}$ is defined as a rate of the maximum magnitude of the roll angle in Phase 1 to the limit magnitude $\phi_{l}$ of that.

$$
\begin{aligned}
C P_{1} & =\frac{(\text { Maximum magnitude of roll angle in Phase } 1)}{(\text { Limit magnitude of roll angle })} \\
& =\frac{\max (|\phi(t)|)}{\phi_{l}} \times 100
\end{aligned}
$$

where the limit magnitude $\phi_{l}$ of rolling angle is empirically fixed on 21 [deg] in this paper. As an evaluation index of the effectiveness in Phase 1, the avoidance risk rate $A_{r r}$ is decided to average those items in Eqs. (2) and (3).

$$
A_{r r}=\frac{C L+C P_{1}}{2}
$$

The efficiency in Phase 1 is quantified with the total helm revolution angle $H R_{1}$, which integrates the magnitude of the instant helm revolution angle $|\dot{\theta}(t)| d t$ in a short time $d t$ from $t=0$ to $t=t_{a}$ as follows.

$$
H R_{1}=\int_{0}^{t_{a}}|\dot{\theta}(t)| d t
$$




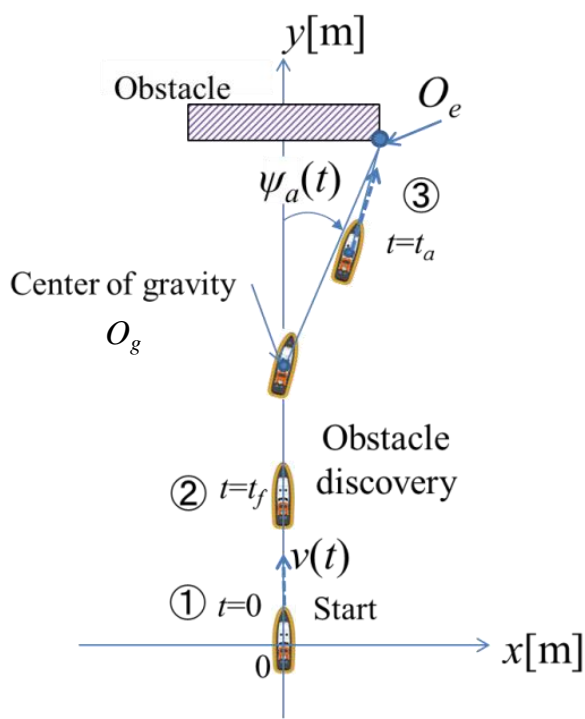

Fig. 6 Outline of collision avoidance in Phase 1

\subsection{Veering Period to Resume Original Course (Phase 2)}

After the moment $t=t_{a}$ of completing avoidance in Phase 1, it shifts to Phase 2. As shown in Fig. 7, Phase 2 is defined for the time from $t=t_{a}$ to $t=t_{r}$ at the moment of resuming the original course as shown in the circled digit five. The condition of resuming the original course is empirically decided that the magnitude of the helm angle $|\theta(t)| \leq \pi[\mathrm{rad}]$ and the magnitude of the course $|\psi(t)| \leq 8[\mathrm{deg}]$.

The effectiveness to resume the original course quickly and safely in Phase 2 is quantified with two items, which are the resuming delay rate $R D$ on the response of resuming the original course and the capsize risk rate $\mathrm{CP}_{2}$ on the safety. The resuming delay rate $R D$ is defined as a rate of the complete resuming time $t_{r}-t_{a}$ to the predicted time to reach the end of the evaluation area at the starting moment of Phase 2, because the completion resuming time is relatively evaluated to the hull speed like the complete avoidance time of Phase 1 in Eq. (2).

$$
\begin{aligned}
R D & =\frac{(\text { Complete resuming time })}{(\text { Predicted time to end of evaluation area at starting moment of Phase })} \times 100 \\
& =\frac{(\text { Complete resuming time })}{\left(\begin{array}{l}
\text { Distance to end of evaluation area } \\
\text { at starting moment of Phase2 }
\end{array}\right) /\left(\begin{array}{c}
\text { Boat speed } \\
\text { at starting moment of Phase 2 })
\end{array}\right.} \times 100 \\
& =\frac{t_{r}-t_{a}}{\left\{\frac{1000-y\left(t_{a}\right)}{\cos \psi\left(t_{a}\right)}\right\} / v\left(t_{a}\right)} \times 100
\end{aligned}
$$

As similar to that defined in Phase 1, the capsize risk rate $C P_{2}$ is defined as a rate of the maximum magnitude of the roll angle in Phase 2 to the limit magnitude $\phi_{l}$ of that.

$$
\begin{aligned}
C P_{2} & =\frac{(\text { Maximum magnitude of roll angle in Phase } 2)}{(\text { Limit magnitude of roll angle })} \times 100 \\
& =\frac{\max (|\phi(t)|)}{\phi_{l}} \times 100
\end{aligned}
$$


As an evaluation index of the effectiveness in Phase 2, the resuming risk rate $R_{r r}$ is decided to average those items in Eqs. (6) and (7).

$$
R_{r r}=\frac{R D+C P_{2}}{2}
$$

As similar to that defined in Phase 1, the efficiency in Phase 2 is quantified with the total helm revolution angle $H R_{2}$, which integrates the magnitude of the instant helm revolution angle $|\dot{\theta}(t)| d t$ in a short time $d t$ from $t=t_{a}$ to $t=t_{r}$ as follows.

$$
H R_{2}=\int_{t_{a}}^{t_{r}}|\dot{\theta}(t)| d t
$$

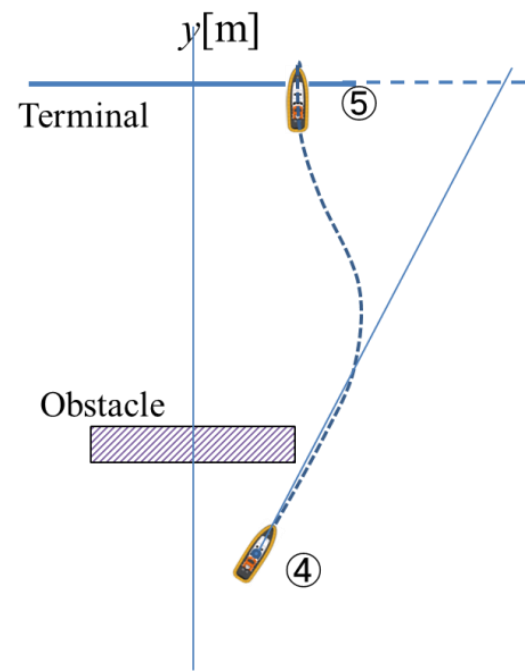

Fig. 7 Outline of resuming original course in Phase2

\subsection{Total Evaluation of Operability}

The task defined in the proposed evaluation method consists of two targets. One of the targets is avoiding the obstacle quickly and safely in Phase 1. Another is resuming the original course quickly and safely in Phase 2. Therefore, the total evaluation of effectiveness and efficiency for the task based on usability is finally evaluated with the combination of the evaluation results for each phase.

The index $A R$ of the effectiveness for each whole simulation is defined as the average of the avoidance risk rate $A_{r r}$ in Eq. (4) and the resuming risk rate $R_{r r}$ in Eq. (8).

$$
A R=\frac{A_{r r}+R_{r r}}{2}
$$

The index $H R$ of the efficiency for each whole simulation is defined as the addition of the total helm revolution angle $H R_{1}$ in Eq. (5) and the total helm revolution angle $H R_{2}$ in Eq. (9).

$$
H R=H R_{1}+H R_{2}
$$

The satisfaction is evaluated by questionnaire of the subjects. It can be said that the operability is good, when those values of $A R$ and $H R$ are small and the satisfaction of subjects is shown by the questionnaire. 


\subsection{Examples of Calculating Evaluation Indexes for Effectiveness and Efficiency}

As examples of calculating the evaluation indexes Eqs. (10) and (11) defined in Section 4.4, two typical cases, such as a task success case shown in Fig. 8 and a task failure case shown in Fig. 9, were provided. The specification of a pleasure boat for these cases is given as follows. The total length is $18.8[\mathrm{~m}]$, the total width is $6.1[\mathrm{~m}]$, the maximum propeller revolution is $2100[\mathrm{rpm}]$, and the maximum speed is $32.0[\mathrm{kt}](=16.5[\mathrm{~m} / \mathrm{s}])$. As the operation condition, the visibility is assumed to be $200[\mathrm{~m}]$, the wind-tidal current is assumed not to exist, and the propeller revolution is assumed to be four respectively fixed patterns at $900[\mathrm{rpm}], 1200[\mathrm{rpm}], 1500[\mathrm{rpm}]$, and 1800 [rpm].

The task failure case is a typical data of an immature subject. For this task failure case, those evaluation indexes should be calculated as comparatively large values corresponding to worse operability, because the immature subject could not accomplish the task effectively and efficiency as shown in Fig. 9. On the other hand, the task success case is a typical data of the same subject but who could master the maneuverability by training the task for a long time. This task success case can be considered to be nearly equal with an ideal case, which immature subjects could effectively and efficiency achieve the task by excellent operability of pleasure boats without any experience. Therefore, those evaluation indexes of the task success case should be calculated as comparatively small values corresponding to good operability.

Table 1 shows the calculation results for the task success case, and Table 2 shows the calculation results for the task failure case. From these tables, it can be seen that the total evaluation indexes $A R$ and $H R$ for the task failure case are larger than those of the task success case as expected. The reason can be explained as follows. As shown in Fig. 9 (c), the immature subject added excessive operation of the helm immediately after finding the obstacle. As a result, the immature subject could avoid the obstacle quickly as shown in Fig. 9 (a), but caused excessive rolling angle of the pleasure boat as shown in Fig. 9 (b). Then, the immature subject could not completely return the helm for preparation of resuming the original course at the complete avoidance moment $t=t_{a}$ as shown in Fig. 9 (c), though the trained subject could do as shown in Fig 8 (c). As a result, winding wakes were caused, and resuming the original course was delayed as shown in Fig. 9 (a).

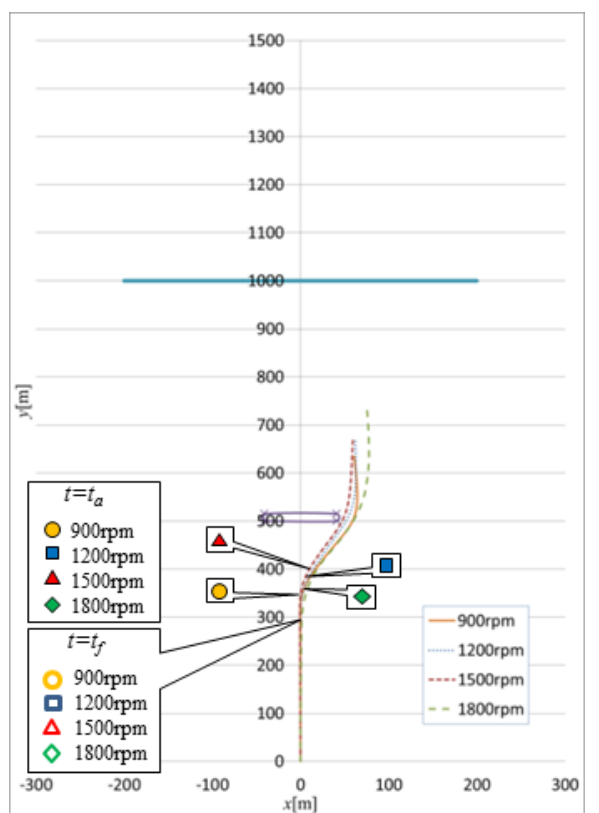

(a) Wake

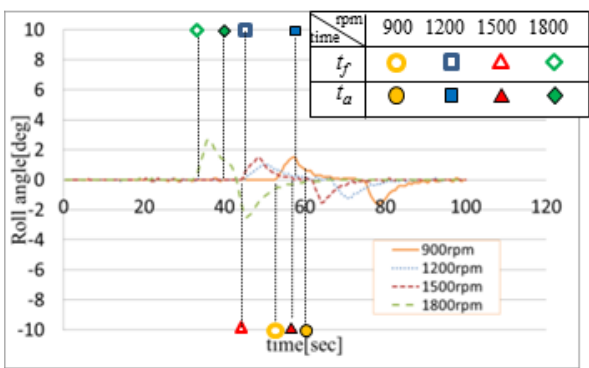

(b) Rolling angle

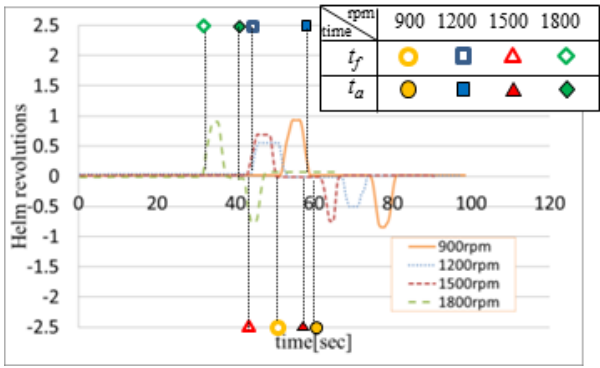

(c) Helm revolutions

Fig. 8 Task success case 


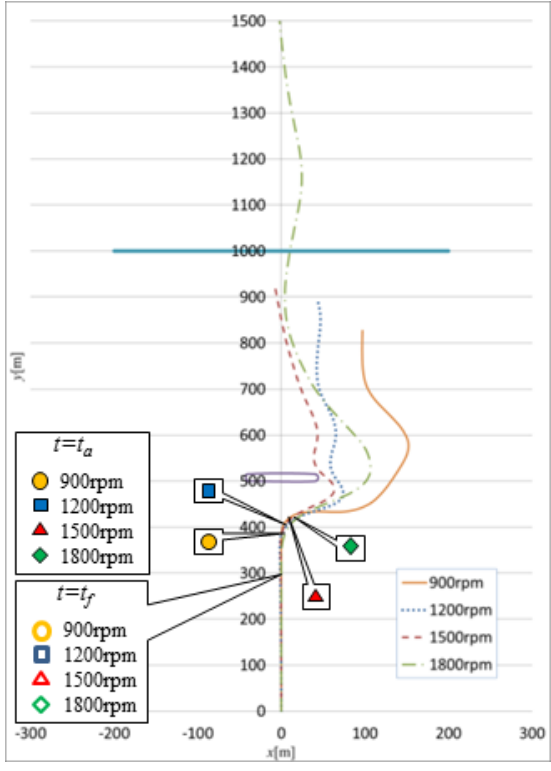

(a) Wake

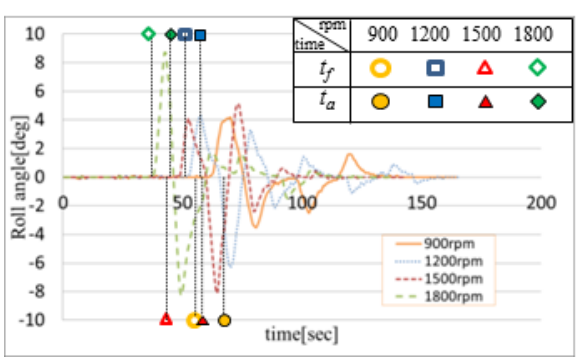

(b) Roll angle

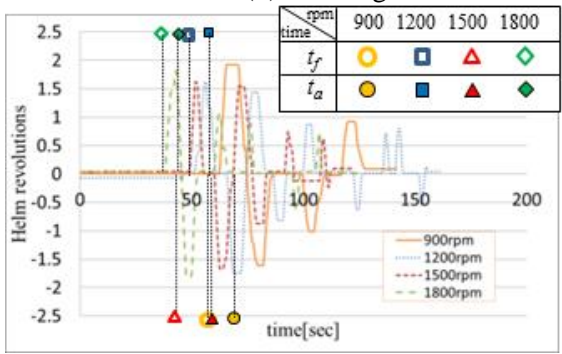

(c) Helm revolutions

Fig. 9 Task failure case

Table 1 Evaluation result for task success case

\begin{tabular}{|c|c|c|}
\hline $\begin{array}{c}\text { Propeller } \\
\text { rev. [rpm] }\end{array}$ & $\begin{array}{c}\text { Effectiveness } \\
A R\end{array}$ & $\begin{array}{c}\text { Efficiency } \\
H R\end{array}$ \\
\hline \hline 900 & 17.3 & 3.4 \\
\hline 1200 & 17.4 & 2.1 \\
\hline 1500 & 19.8 & 2.8 \\
\hline 1800 & 19.4 & 3.6 \\
\hline \hline Average & 18.5 & 3.0 \\
\hline
\end{tabular}

Table 2 Evaluation result for task failure case

\begin{tabular}{|c|c|c|}
\hline $\begin{array}{c}\text { Propeller } \\
\text { rev. [rpm] }\end{array}$ & $\begin{array}{c}\text { Effectiveness } \\
A R\end{array}$ & $\begin{array}{c}\text { Efficiency } \\
H R\end{array}$ \\
\hline \hline 900 & 30.9 & 10.7 \\
\hline 1200 & 32.8 & 17.7 \\
\hline 1500 & 38.4 & 14.1 \\
\hline 1800 & 53.5 & 12.9 \\
\hline \hline Average & 38.9 & 13.8 \\
\hline
\end{tabular}

Additionally, the average 18.5 of the effectiveness $A R$ and the average 3.0 of the efficiency $H R$ in Table 1 are decided as each standard value of the operability evaluation for manual hydraulic steering system as shown in the next section.

\section{Simulation of Operability Evaluation for Manual Hydraulic Steering System}

\subsection{Simulation Method}

For ten subjects, the simulations were planned in order of a guidance of the simulation, a trial operation for a test course, the simulation of operability evaluation, and the questionnaire. In the simulation guidance, the simulation object, the outline of the manual hydraulic steering system, the turning principle of pleasure boats, the simplified ship simulator, and the simulation task are explained. In the trial operation, the subjects can try to operate the pleasure boat through the narrow and winding trial course for ten minutes, and they can experience the maneuverability of the pleasure boat before the evaluation simulation. In the evaluation simulation, the course shown in Fig. 4 and two visibility conditions, such as 300 [m] and 200 [m], are assumed. In the visibility condition 300 [m], the subjects can avoid the obstacle smoothly, since the distance to the obstacle is still 
enough at the moment of finding the obstacle. In the visibility $200[\mathrm{~m}]$, they should avoid it emergently. The wind-tidal current is assumed not to exist, and the propeller revolution is assumed to be four respectively fixed patterns at 900 [rpm], 1200 [rpm], 1500 [rpm], and $1800[\mathrm{rpm}]$. As the pleasure boat used in the trial operation and in the evaluation simulation, the same one as used in Section 4.4 is assumed. As the ten subjects of the simulation, eight students and two staffs at Nagaoka National College of Technology were selected. All subjects have no permit of boat's operator, and nine of them have driving license of automobiles. The order of the experiment for each condition was randomly determined so that the effects might be negated as much as possible.

\subsection{Evaluation Results of Safe Avoidance in Visibility 300 [m]}

Figure 10 shows the evaluation results of the effectiveness in the case of the visibility 300 [m]. From Fig. 10 (a) to show the evaluation results for each subject, it can be seen that all subjects have a tendency to increase the index value on the standard one as the propeller revolution increases. From Fig. 10 (b) to show the average and the standard deviation for each propeller revolution condition, it can be seen that the result seems to have no extreme increase of the index value on the standard one. Figure 11 shows the evaluation results of the efficiency in the case of the visibility 300 [m]. From Fig. 11 (a) and (b), it can be seen that the results have a trend to decrease the index value as the propeller revolution increases, though the results have a little difference for each subject. This trend is caused by rise of the normal pressure $P_{N}$ for the increase of the propeller revolution, which can make the fluid velocity $V$ of Eq. (1) accelerate. As a result, the rudder angle $\delta$ can be made smaller for generating the same magnitude of the normal pressure $P_{N}$, and the total helm operation can be made a little. Also almost answers of the questionnaires, which could be gotten by 8 subjects, show that they did not feel difference of the operability against change of the propeller revolution, and they could operate safely. These results show that the index of the efficiency can be improved as the propeller revolution increases if subjects have some spare time to avoid the obstacle, and the steering system shows good operability, which does not depend on the propeller revolution condition, in the safe avoidance of the visibility $300[\mathrm{~m}]$.

\subsection{Evaluation Results of Emergency Avoidance in Visibility 200 [m]}

Figure 12 shows the evaluation results of the effectiveness in the case of the visibility 200 [m]. From Fig. 12 (a) for each subject, it can be seen that the index values steeply rise on the standard one. Especially, the average of the index in Fig. 12 (b) notably rises as the propeller revolution increases. Simultaneously, the standard deviation rises, since the index value of the subjects No. 6, No. 7, and No. 8 seems not to increase for raise of the propeller revolution as shown in Fig. 12 (a). This trend could show the difference of the operation skill between the subjects. Figure 13 shows the evaluation results of the efficiency in the case of the visibility 200 [m]. From Fig. 13 (a) for each subject, the index value mostly grows on the standard one, which is considered as ideal. The standard deviation of the index value shown in Fig. 13 (b) increases as the contrary trends of the visibility 300 [m] in Section 5.2, since the helm operations are made more than necessary. Table 3 shows the questionnaire result on satisfaction for the subjects. From the results for the questions Q1, Q2 and Q3, it can be seen that most of them felt the difference in the operability accompanying the rise of the number of propeller revolutions. From the results for the question Q4 and Q5, it can be seen that almost all of them needed to confirm the number of helm revolutions with the rudder angle meter, and that all of them felt the difficulty for the operability of the pleasure boat compared with that of cars. These results show that the operability of the steering system gets worse as the propeller revolution increases in the emergency avoidance of the visibility $200[\mathrm{~m}]$. 


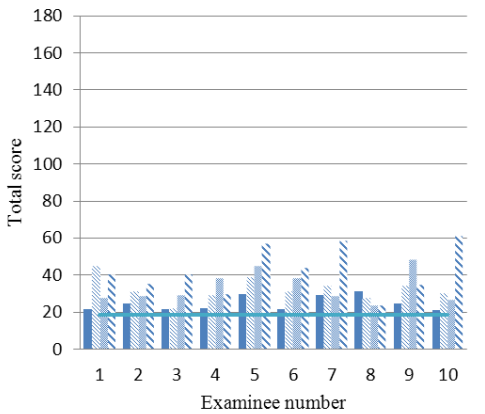

(a) Evaluation results of each examinee

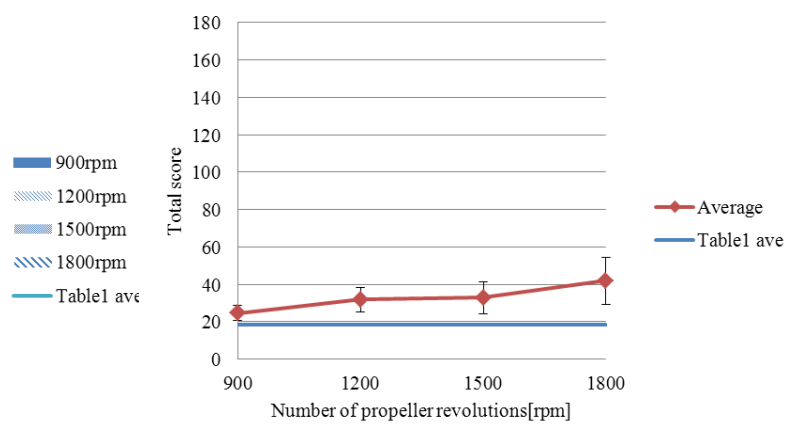

(b) Average and standard deviation

Fig. 10 Evaluation results of effectiveness for visibility $300 \mathrm{~m}$

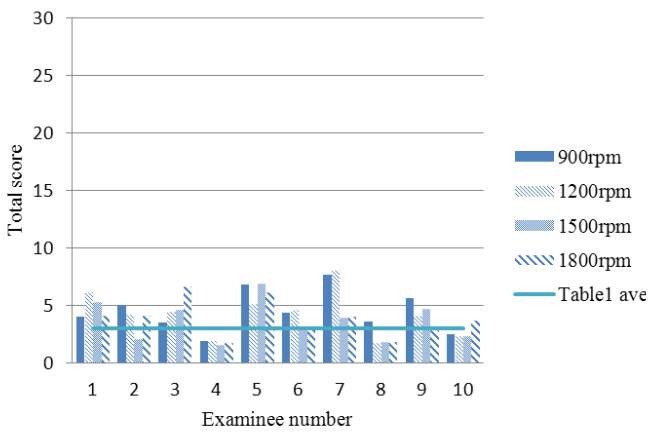

(a) Evaluation results of each examinee

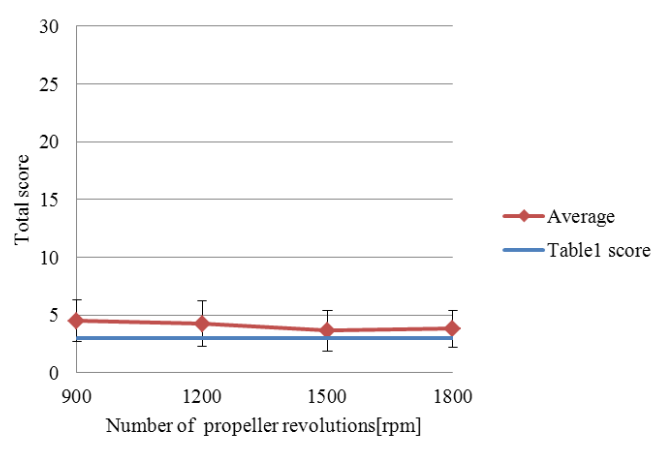

(b) Average and standard deviation

Fig. 11 Evaluation results of efficiency for visibility $300 \mathrm{~m}$

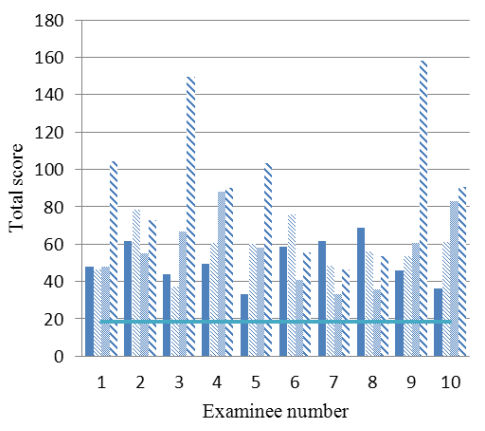

(a) Evaluation results of each examinee

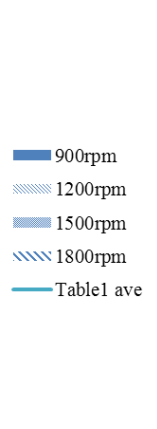

Fig. 12 Evaluation results of effectiveness for visibility $200 \mathrm{~m}$

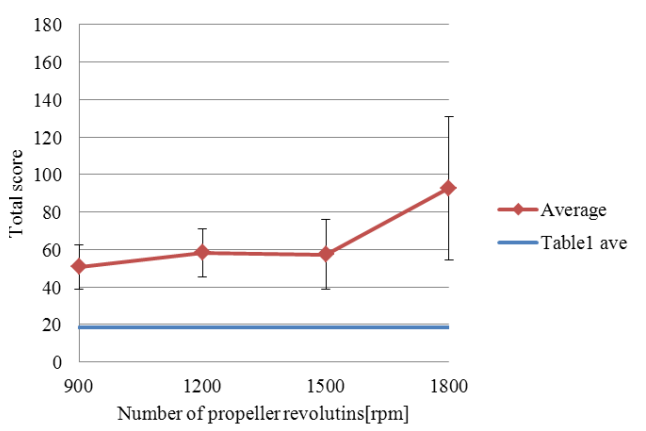

(b) Average and standard deviation

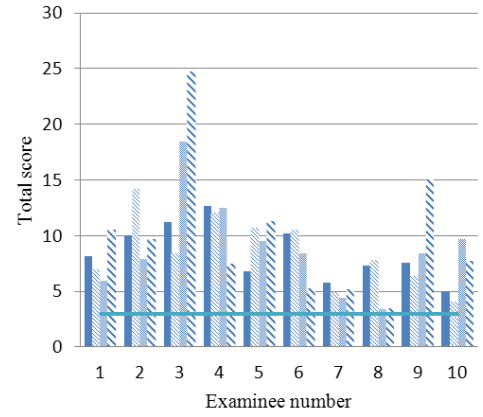

(a) Evaluation results of each examinee

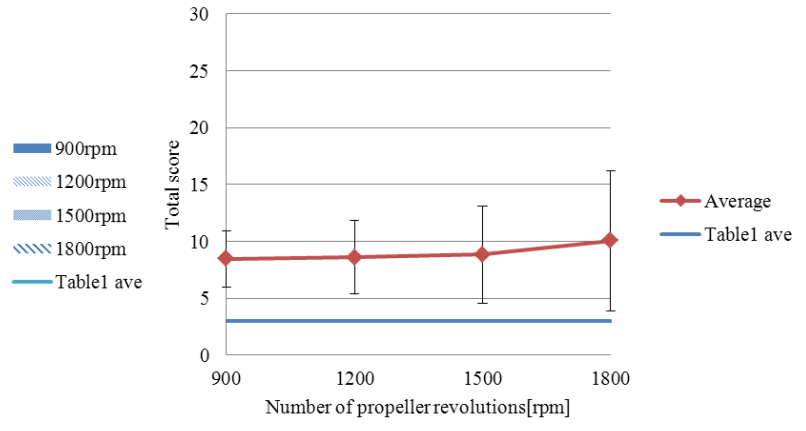

(b) Average and standard deviation

Fig. 13 Evaluation results of efficiency for visibility $200 \mathrm{~m}$ 
Table 3 Questionnaire result on satisfaction

\begin{tabular}{|c|l|c|c|c|c|c||c|c|}
\hline No. & \multicolumn{1}{|c|}{ Question } & $\begin{array}{c}\text { Strongly } \\
\text { agree }\end{array}$ & Agree & $\begin{array}{c}\text { Neither } \\
\text { agree nor } \\
\text { disagree }\end{array}$ & Disagree & $\begin{array}{c}\text { Strongly } \\
\text { disagre }\end{array}$ & Average & $\begin{array}{c}\text { Standard } \\
\text { devision }\end{array}$ \\
\hline \hline Q1 & I could operate as I intended. & 5 & 4 & 3 & 2 & 1 & $\mathbf{3 . 6}$ & $\mathbf{0 . 7 0}$ \\
\hline Q2 & $\begin{array}{l}\text { It was easy to operate when the number of } \\
\text { propeller revolutions rose. }\end{array}$ & 5 & 4 & 3 & 2 & 1 & $\mathbf{1 . 9}$ & $\mathbf{0 . 8 8}$ \\
\hline Q3 & $\begin{array}{l}\text { I felt the difference in the operability by } \\
\text { the number of propeller revolutions. }\end{array}$ & 5 & 4 & 3 & 2 & 1 & $\mathbf{4 . 6}$ & $\mathbf{0 . 7 0}$ \\
\hline Q4 & $\begin{array}{l}\text { I often needed to confirm the number of } \\
\text { helm revolutions with the rudder angle }\end{array}$ & 5 & 4 & 3 & 2 & 1 & $\mathbf{4 . 7}$ & $\mathbf{0 . 4 8}$ \\
\hline Q5 & $\begin{array}{l}\text { It was hard to operate the pleasure boat } \\
\text { compared with cars. }\end{array}$ & 5 & 4 & 3 & 2 & 1 & $\mathbf{5 . 0}$ & $\mathbf{0 . 0 0}$ \\
\hline
\end{tabular}

\subsection{Design Criteria of Electronic Controlled Steering System}

Figure 14 (a) and (b) show the worst evaluation result of the subject No. 3, which are classified by each phase. From Fig. 14, it can be seen that the ratio of Phase 2 becomes larger than that of Phase 1 as the propeller revolution increases. A reason of the trend is that the winding wakes shown in Fig. 14 (c) are caused by the swing operation of the helm shown in Fig. 14 (e). As a result, resuming the original course is made late and excessive rolling angle occurs as shown in Fig. 14 (d). That is why the evaluation value got worse.

Next, the reason for the swing operation of the helm should be analyzed. As an example data for the analysis, Fig. 15 shows both the time history response of the helm revolution and the course of the pleasure boat for the subject No. 3 in the condition such as the visibility $200[\mathrm{~m}]$ and the propeller revolution $1800[\mathrm{rpm}]$. From this figure, it can be seen that the dynamics of the pleasure boat makes the response of the course late for the helm operation. If the course at the complete avoidance time $t=t_{a}$ was kept after that, it could be possible to resume the original course quickly in Phase 2. However, the actual course in the first stage of Phase 2 continued to change into the avoidance, that is, the overshoot of the course occurred since the subject operated the helm more than necessary in the closing stage of Phase 1. Resuming the original course from the overshoot in the first stage of Phase 2, the subject also operated more than necessary in a short time as the circled parts shown in Fig. 15, since the subject could not estimate the normal pressure $P_{N}$ and could not consider the dynamics of the pleasure boat accurately. Especially, in the condition of the propeller revolution $1800[\mathrm{rpm}]$, the dynamics of the pleasure boat caused such notable winding wakes, since the magnitude of the normal pressure $P_{N}$ becomes large sharply as rise of the flow velocity $V$ as show in Eq. (1) and the sensitivity of the hull motion to the helm operation gets higher.

These results show that it is difficult for captains to grasp some information, such as the magnitude of the normal pressure $P_{N}$ that nonlinearly changes against the rudder angle and the propeller revolution, through manual hydraulic steering systems. Also, they show that the dynamics of pleasure boats could cause excessive rolling angle and winding wake in particular situations, such as emergency avoidance which are necessary to operate in short time, if the propeller revolution was relatively high. The electronic controlled steering system, which is proposed to improve such operability in Ref. (3), has some functions such as transferring arbitrary reaction torque to captains through the helm and designing arbitrary transfer characteristics from the helm operation to the rudder angle with by-wire technique. One of the design criteria for such steering system is to show the magnitude of the normal pressure $P_{N}$, which could be estimated by some algorithm, into captains with reaction torque of the helm operation, and to make low pass filter, which should be designed for the dynamics of the pleasure boat, of the transfer characteristics from the helm operation to the rudder angle so that large swing operation is not generated. 


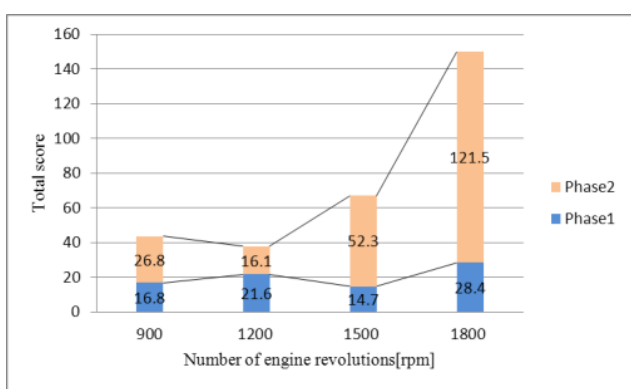

(a) Evaluation results of effectiveness

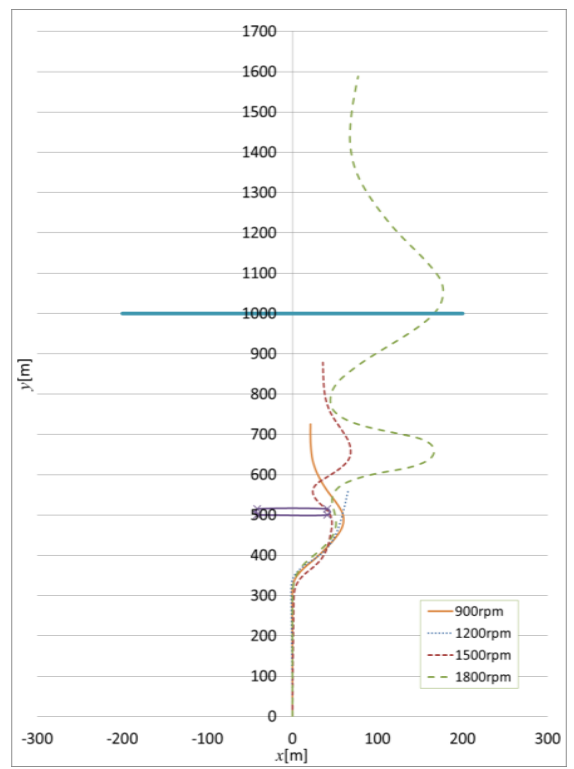

(c) Wake

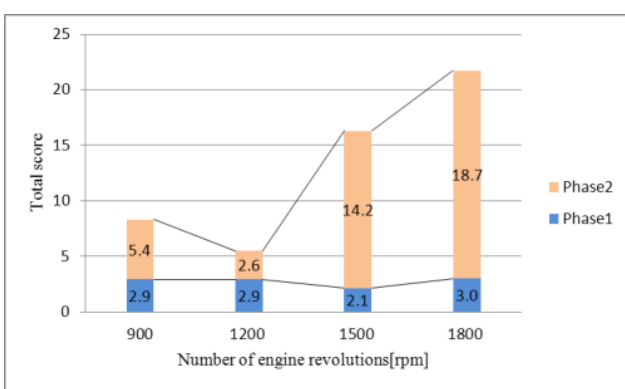

(b) Evaluation results of efficiency

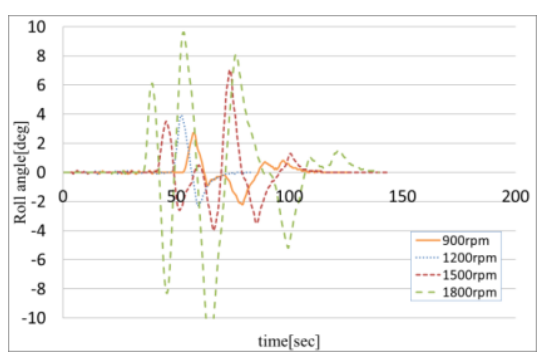

(d) Rolling angle

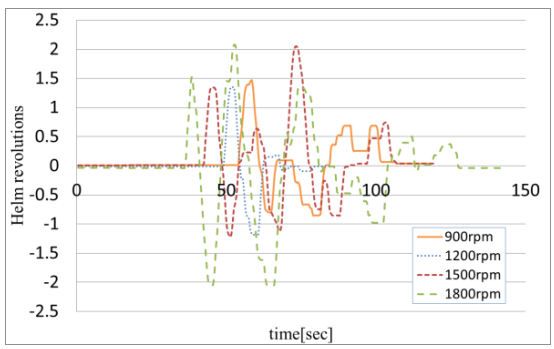

(e) Helm revolutions

Fig. 14 Evaluation results of examinee 3 for visibility $200 \mathrm{~m}$

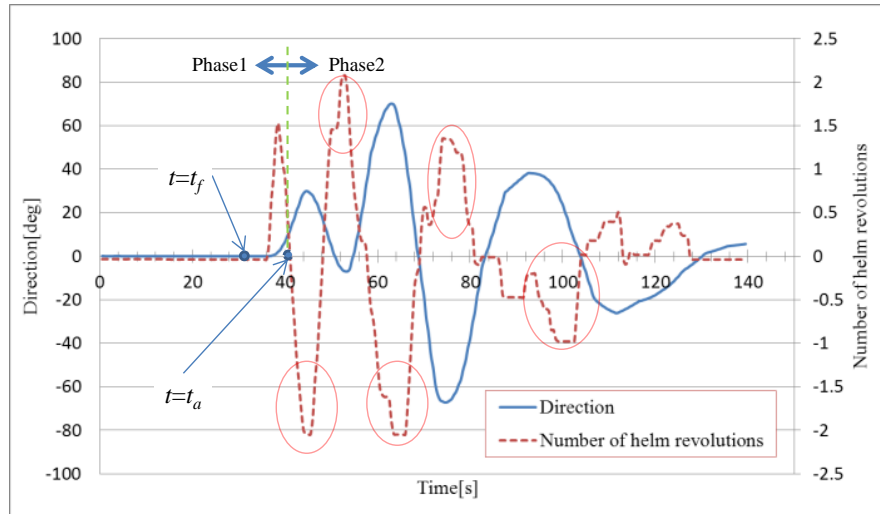

Fig. 15 Behavior of pleasure boat at propeller revolution $1800 \mathrm{rpm}$ in visibility $200 \mathrm{~m}$

\section{Conclusion}

First, this paper proposed an evaluation method of the operability for steering systems of pleasure boats. Based on the usability as addressed by ISO 9241-11, the task of the evaluation method is defined as avoiding an obstacle and resuming the original course in poor visibility. A total index of the effectiveness including the complete time of avoiding obstacle, the complete time of resuming the original course and the rolling angle is defined, and a total index of the efficiency including the total revolution of helm is defined. Some 
example of calculating those indexes were shown for the typical data of both the task success case and the task failure case.

Next, a manual hydraulic steering system is evaluated with the proposed method. As a result, the following knowledge is given.

1) In case of safe avoidance, the manual hydraulic steering system shows a satisfactory operability to achieve the task regardless of the propeller revolution.

2) In case of emergency avoidance, the operability of the manual hydraulic steering system gets worse as rise of the propeller revolution, and brings excessive rolling angle and winding wakes.

This knowledge suggests some functions of the electronic control steering system, such as to show captains information on the estimated normal pressure with the reaction torque of helm operation, and such as to have some frequency characteristics from the helm operation to the rudder angle. The frequency characteristics should be designed for the dynamics of pleasure boats.

\section{Acknowledgement}

This study was supported by the research grant of Uniontool Scholarship Society 2012, for which the authors express their deepest gratitude. And the authors would like to thank the anonymous reviewers for their valuable comments and suggestions to improve the quality of the paper.

\section{References}

(1) Cabinet Office of Japan, Maritime Accident Trends, White Paper on Traffic Safety in Japan 2012, (2013), pp.105-110.

(2) Ikeda, F., Toyama, S., Seta, H. and Ezaki, N., Improvement of Steering Feeling on Maneuverability for Pleasure Boat, Journal of System Design and Dynamics, Vol. 5, No. 5 (2011), pp. 1119-1126.

(3) Toyama, S., Ikeda, F. and Seta, H., A Sliding Mode Controller of Rudder Angle Servomechanism in Steer-by-Wire System of Pleasure Boat, Journal of System Design and Dynamics, Vol. 5, No. 5 (2011), pp. 1127-1140.

(4) Aoyama, M. and Kanno, T., Information and Communication Technologies for Flight Control and Management Systems, The journal of the Institute of Electrical Engineers of Japan, Vol. 123, No. 6 (2003), pp. 339-343. (in Japanese)

(5) Kamogawa, T. and Maekawa, A., Lateral Control Augmentation for Aircraft Using Adaptive Fuzzy Neural Networks, Proceedings of 59th annual meeting of Tokai Branch, Japan Society of Mechanical Engineers, (2010), pp.11-12. (in Japanese)

(6) Kato, A., Usami, N. and Okude, M., Improvement of maneuverability of airplanes with neural network, Proceedings of Environmental and Human Science, Graduate School of Environmental and Human Science, Meijo University, No. 4 (2004), pp. 63-75. (in Japanese)

(7) Kojima, T., Improvement of the Handling Qualities during Hover in the Night or Poor Visibility, Mitsubishi Heavy Industries Technical Review, Vol. 45, No. 4 (2008), pp. 70-73. (in Japanese)

(8) Ema, T. and Mizukura, Y., A Study of Evaluation Method for Pilot's Maneuverability, Scientific and engineering reports of the National Defense Academy, Vol. 38, No. 3 (1991), pp. 223-229. (in Japanese)

(9) Goto, N., Aircraft Handling Qualities and Evaluation, Design Engineering, Vol. 34, No. 6 (1990), pp. 175-179. (in Japanese)

(10) Kobayashi, O., Flying Qualities of Aircraft and Pilot Evaluation, Equilibrium research, 
Vol. 61, No. 4 (2002), pp. 193-198. (in Japanese)

(11) Abe, M., Automotive Vehicle Dynamics Theory and Applications, (2008), pp. 245-248, Tokyo Denki University Press. (in Japanese)

(12) Honda, K., An Outline of Maneuvering Ships, (2008), pp.10-12, Seizando-Shoten Publishing. (in Japanese)

(13) Kurosu, M., Usability Testing, (2003), pp.75, Kyoritsu Shuppan Co., Ltd. (in Japanese) 